\title{
Badis dibruensis, a new species (Teleostei: Badidae) from northeastern India
}

\author{
Kh. Geetakumari ${ }^{1} \&$ W. Vishwanath ${ }^{2}$ \\ 1,2 Department of Life Sciences, Manipur University, Canchipur, Imphal, Manipur 795003, India \\ Email: ${ }^{1}$ geetameme@gmail.com, ${ }^{2}$ wvnath@gmail.com (corresponding author)
}

Date of publication (online): 26 January 2010 Date of publication (print): 26 January 2010 ISSN 0974-7907 (online) | 0974-7893 (print)

Editor: Rema Devi

Manuscript details:

Ms \# 02156

Received 10 March 2009

Final received 14 August 2009

Finally accepted 05December 2009

Citation: Geetakumari, Kh. \& W. Vishwanath (2010). Badis dibruensis, a new species (Teleostei: Badidae) from northeastern India. Journal of Threatened Taxa 2(1): 644-647.

Copyright: ๑ Kh. Geetakumari \& W. Vishwanath 2010. Creative Commons Attribution 3.0 Unported License. JoTT allows unrestricted use of this article in any medium for non-profit purposes, reproduction and distribution by providing adequate credit to the authors and the source of publication.

Author Details: KH. Geetakumari is a research scholar in the Department of Life Sciences, Manipur University. She is pursuing research in Phylogenetic studies of fishes belonging to the order Perciformes found in northeastern India under the supervision of W. Vishwanath.

W. VISHWANATH is a professor in the Departmen of Life Sciences, Manipur University. His field of specialization is Fish and Fisheries. $\mathrm{He}$ is at present engaged in taxonomy and systematics of freshwater fishes of northeastern India

Author Contribution: The study: Kh.G Undergoing research in the Perciformes fishes of northeastern India. WV Supervision of taxonomy and phylogeny of freshwater fishes o northeastern India. Current paper: As a part of the research work, all the badid fishes of the region have been studied and the species is found to be new. Supervised the work and helped in identifying the species.

Acknowledgements: The first author is gratefu to Manipur University for award of UGC research scholarship and the second, to Ministry of Environment and Forests, Govt. of India for financial assistance (Project No. 14/11/2006-ERS/ $\mathrm{RE})$. We thank Mr. Santosh for the collections.
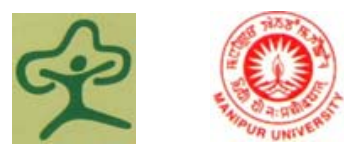

(ே)

OPEN AGGESS I FREE DOWNLOAD
Abstract: A new species of the genus Badis Hamilton is described from Dibru River, Dibrugarh, Brahmaputra basin in Assam, India. The species has the following combination of characters: a conspicuous black blotch covering the superficial part of the cleithrum above pectoral fin base, a small oval-shaped black blotch on the middle of caudal fin, two predorsal bones, interorbital width 9.9-15.0, upper jaw length 6.1-6.9, lower jaw length 7.1-8.3 and orbital diameter 7.6-9.4\% SL. The species differs from its nearest congeners, $B$. badis, $B$. kanabos and $B$. tuivaiei by the absence of dark black or brown vertical bars on sides. A key to species of Badis of India is provided.

Keywords: Assam, Brahmaputra basin, new fish, Perciformes.

\section{INTRODUCTION}

Hamilton (1822) described Labrus badis and L. dario from Gangetic provinces. Bleeker (1854) established genus Badis for L. badis under family Nandidae. Barlow et al. (1963) erected a new family, Badidae, in which Badis has been incorporated based on osteological characters. The genus is separated from the nandid genus Nandus in having a smooth (versus serrated) preopercular margin and edentulous (versus toothed) tongue. As per Kullander \& Britz (2002) the genus is characterized by a combination of the following features: opercle with a single sharp spine at its posterodorsal corner; spinous and soft dorsal fins contiguous, the base of the soft part longer than that of the spinous part; anal fin with three spines; lateral line pores tubed and interrupted; jaws, vomer and palatines with villiform teeth; scales both ctenoid and cycloid; 2-4 dentary foramina; hypobranchial 3-toothed; males with short pelvic fin, not reaching the first dorsal spine; short dorsal fin lappets; rounded caudal fin.

Ahl (1937) described Badis badis assamensis from Assam, while Talwar \& Jhingran (1991) did not recognize any subspecies of B. badis. Kullander \& Britz (2002) treated $B$. assamensis as a valid species and designated a neotype. They also described $B$. blosyrus and B. kanabos from Brahmaputra basin, Assam and B. ferrarisi from Myanmar. Vishwanath \& Shanta (2004) described B. tuivaiei from Tuivai River in Brahmaputra basin. Vishwanath et al. (2007) also recorded $B$. ferrarisi from Chindwin basin in Manipur. Thus, six species of Badis are currently recognized from northeast India.

During field surveys in northeast India in 2006 and 2007, specimens of an undescribed Badis were collected from Dibru River, Dibrugarh, Brahmaputra basin, Assam. The species is herein described as Badis dibruensis sp. nov.

\section{Materials and Methods}

Measurements were made point to point with dial calipers to the nearest $0.1 \mathrm{~mm}$ and expressed as percentages of standard length (SL). Counts and measurements were made on the left side of specimens under a PC-based binocular stereo zoom microscope (Olympus SZ40) with transmitted light. Counts and measurements followed Kullander \& Britz (2002). The method of clearing and staining of specimens for osteology follows Hollister (1934). Identification and nomenclature of bones and vertebral counts follows Greenwood (1976). For branchial toothplate count, the first gill arch on the left side of the specimens was taken, and plates starting from hypobranchial to epibranchial of the outer side were counted. Type specimens are deposited in the Manipur University Museum of fishes (MUMF). 
Badis dibruensis sp. nov.

(Images 1\& 2)

\section{Type material}

Holotype: $37.3 \mathrm{~mm}$ SL, 16.iii.2006, 27³2'09.98”N \& 94⒌'02.31"E, Dibru, Brahmaputra drainage, Dibru River, Dibrugarh, Assam, India; coll. Santosh, MUMF-Per 95.

Paratypes: MUMF-Per 96-110, 15, 23.0-40.8mm SL, data as for holotype; MUMF Per-108-110, 3, dissected, cleared and stained for osteology.

\section{Diagnosis}

Badis dibruensis sp. nov. is distinguished from its nearest congener, $B$. badis in having two (vs. three) predorsal bones; small oval (vs. large rectangular) black blotch in the mid-base of caudal fin; more interorbital width (9.9-15.0 vs. $6.5-8.3 \%$ SL); shorter upper jaw (6.1-6.9 vs.8.2-10.2\% SL) and lower jaw (7.1-8.3 vs. $11.3-14.5 \% \mathrm{SL})$. It is distinguished from $B$. kanabos in having smaller eye diameter (7.6-9.4 vs. 9.5-12.7\% $\mathrm{SL})$. It is also distinguished from $B$. tuivaiei in having vertebrae 27 (vs. 31); more interorbital width (9.9-15.0 vs. 5.6-7.2\% SL); shorter upper jaw (6.1-6.9 vs. 8.5-10.4\% SL). B. dibruensis differs from all three mentioned species by the absence (vs. presence) of dark black or brown vertical bars on sides.

\section{Description}

Morphometric data and counts are in Tables 1 \& 2 , respectively. Frequency distributions of meristic characters are provided in Table 3 and comparison with related species in Table 4. Body elongate, moderately compressed laterally. Predorsal profile in small specimens straight, sloping at some angle as of prepelvic profile in larger specimens and more strongly as the size increases. Orbit situated in anterior half of head, at about mid-lateral axis of body. Jaws almost equal anteriorly, lower jaw slightly projecting, maxilla reaching to 1/ 3 of orbit. Opercular spine slender, with a sharp tip. Palatine, vomer and parasphenoid toothed.

Pores: dental 3, anguloarticular 2, preopercular 6, nasal 6, supraorbital 3 , extrascapular 5 , supracleithral 2 , posttemporal 2 , coronalis 1, lachrymal 3, infraorbital pores 3-4. A row of free neuromasts extending across gap between lachrymal and anteriormost infraorbital.

Scales strongly ctenoid on sides, cycloid on top of head. Predorsal scales anterior to coronalis pore 4-5, posteriorly 89. Scales on cheek 3 , mostly ctenoid, a few scales anterior to cheek cycloid. Circumpeduncular scale rows 10 above, 10 below lateral line, totaling 22 . Scales in vertical row $1 \frac{1}{2}$ above, 7 below lateral lines. Vertebra 27 (15/12).

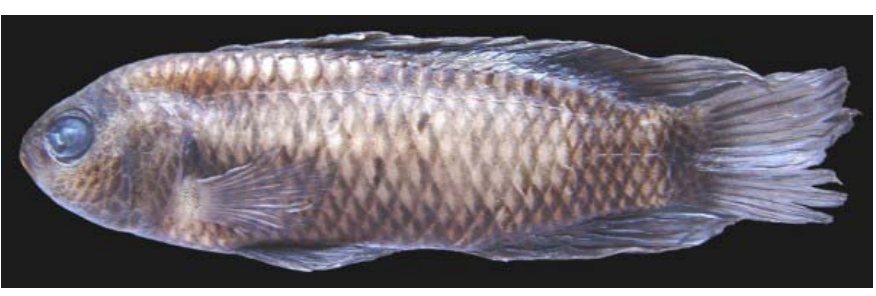

Image 1. Side view of Badis dibruensis sp. nov., holotype, adult male, MUMF-Per 95, 39.3mm SL

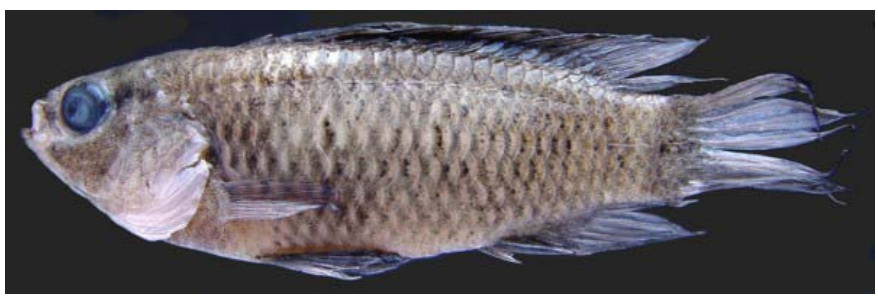

Image 2. Side view of Badis dibruensis sp. nov., paratype, adult female, MUMF-Per 96, 37.3mm SL

Soft dorsal and anal fins with rounded tips reaching to almost about $1 / 3$ or $1 / 4$ of caudal fin. Caudal fin rounded. Pectoral fin rounded, extending about $2 / 3$ distance to anal-fin origin. Pelvic fin pointed, inner branch of second soft ray longest, not reaching up to vent. Orbital diameter, interorbital width and upper jaw length respectively (7.69.4), (9.9-15.0) and $(7.1-8.3) \%$ SL.

\section{Colouration}

In $10 \%$ formaline, overall dark brownish to yellowish. Preorbital stripe dark grey, continued across chin; postorbital stripe blackish, formed by a single blotch close to orbit; dark pigment also on one scale posterior to that blotch. No supraorbital stripe. A conspicuous black blotch covering the superficial part of the cleithrum above pectoral-fin base. Dorsal fin dusky. Dorsal lappets white along distal margin, bordered by blackish submarginal stripe in male. Caudal fin with a small oval black blotch at middle of base, also covering last lateral scale on body. Anal fin dusky basally, soft part hyaline throughout in large specimens. Pelvic fin dusky in small specimens, dark brown in large specimens. Vertical dark black or brown bars are completely absent across the side and caudal peduncle.

Table 1. Proportional measurements of Badis dibruensis sp. nov. in percentage of standard length except standard length.

\begin{tabular}{|c|c|c|c|c|c|c|c|c|c|}
\hline \multirow[b]{2}{*}{ Proportional Measurements } & \multirow[t]{2}{*}{ Holotype } & \multicolumn{4}{|c|}{$\begin{array}{c}\text { Paratypes } \\
\mathrm{N}=4\end{array}$} & \multicolumn{4}{|c|}{$\begin{array}{c}\text { Paratypes } \\
\mathrm{N}=10\end{array}$} \\
\hline & & Mean & Min. & Max. & S.D. & Mean & Min. & Max. & S.D. \\
\hline Head length & 29.3 & 31.7 & 26.5 & 36.5 & 5.0 & 30.1 & 26.5 & 36.5 & 3.3 \\
\hline Snout length & 6.6 & 7.5 & 6.8 & 8.3 & 0.7 & 6.8 & 5.4 & 8.3 & 0.9 \\
\hline Orbital diameter & 7.6 & 9.0 & 8.7 & 9.7 & 0.6 & 8.6 & 7.6 & 9.4 & 0.7 \\
\hline Lower jaw length & 7.9 & 8.1 & 7.8 & 8.3 & 0.3 & 7.8 & 7.1 & 8.3 & 0.4 \\
\hline Body depth & 29.5 & 28.3 & 25.4 & 30.0 & 2.5 & 29.6 & 25.4 & 30.1 & 1.9 \\
\hline Pelvic fin length & 27.5 & 24.8 & 22.6 & 27.4 & 2.4 & 25.9 & 22.6 & 27.5 & 1.8 \\
\hline Pelvic to anal fin distance & 30.5 & 31.5 & 26.1 & 35.8 & 4.9 & 31.8 & 26.1 & 35.8 & 3.6 \\
\hline
\end{tabular}


Table 2. Counts of Badis dibruensis sp. nov. In case of paratypes, maximum and minimum numbers are given.

\begin{tabular}{llll}
\hline Counts & Holotype & Min. & Max. \\
\hline D & $16 / 8$ & $15 / 8$ & $17 / 8$ \\
$\mathrm{P}$ & 14 & 13 & 14 \\
$\mathrm{~A}$ & $\mathrm{iii}, 6$ & 5 & 8 \\
Lateral scale rows & 27 & 25 & 29 \\
Lateral scale count & $20 / 4$ & $19 / 4$ & $22 / 5$ \\
Lateral transverse scales & $11 / 2 / 1 / 6$ & $11 / 2 / 1 / 6$ & $11 / 2 / 1 / 6$ \\
Circumpeduncular scales & 22 & 21 & 22 \\
Toothplate count & & 7 & 7 \\
vertebrae & & 27 & 27 \\
\hline
\end{tabular}

\section{Sexual dimorphism}

Males have uniform dark brown fins with white dorsal fin lappets along distal margin, bordered by blackish submarginal stripe. Extension of the posteriormost tip of the dorsal fin extends upto $1 / 2$ of the caudal fin.

In females, body pale yellow with dark grey spots, fins with uniform light brown coloration. Extension of the posteriormost tip of dorsal fin extends upto $1 / 3$ of the caudal fin.

\section{Etymology}

Named after the Dibru River, the type locality.

\section{Distribution}

Presently known from Dibru River at Dibrugarh, Assam, Brahmaputra drainage (Fig. 1)

\section{Discussion}

Badis dibruensis is similar to $B$. badis, $B$. kanabos and $B$. tuivaiei in having a conspicuous dark blotch covering the superficial part of cleithrum above pectoral-fin base. However, the species differs from them in morphometric characters as in diagnosis above. $B$. dibruensis is distinct from $B$. assamensis and $B$. blosyrus, other two congeners of Brahmaputra basin in absence (vs. presence) of blotch on opercle.

Five species of Badis, viz., B. corycaeus, B. ferrarisi, B.
Table 3. Frequency distribution of meristic characters

\begin{tabular}{|c|c|c|c|c|c|}
\hline \multicolumn{6}{|c|}{ a. Dorsal fin counts (spines/soft rays) } \\
\hline $\begin{array}{l}\text { Counts } \\
\text { Specimens }\end{array}$ & $\begin{array}{l}15 / 8 \\
1\end{array}$ & $\begin{array}{l}16 / 8 \\
8\end{array}$ & $\begin{array}{l}16 / 9 \\
3\end{array}$ & \multicolumn{2}{|l|}{$\begin{array}{l}17 / 8 \\
3\end{array}$} \\
\hline \multicolumn{6}{|c|}{ b. Anal fin counts } \\
\hline Counts & 5 & 6 & 7 & 8 & \\
\hline Specimens & 2 & 7 & 3 & 3 & \\
\hline \multicolumn{6}{|c|}{ c. Pectoral fin counts } \\
\hline Counts & 13 & 14 & & & \\
\hline Specimens & 4 & 11 & & & \\
\hline \multicolumn{6}{|c|}{ d. Lateral scale rows } \\
\hline Counts & 25 & 26 & 27 & 28 & 29 \\
\hline Specimens & 1 & 3 & 9 & - & 2 \\
\hline \multicolumn{6}{|c|}{ e. Lateral line scale counts (upper/lower scales) } \\
\hline Counts & $19 / 4$ & $20 / 4$ & $21 / 4$ & $21 / 5$ & $22 / 5$ \\
\hline Specimens & 1 & 10 & 1 & 1 & 2 \\
\hline \multicolumn{6}{|c|}{ f. Tooth plate } \\
\hline Counts & 6 & 7 & & & \\
\hline Specimens & 1 & 2 & & & \\
\hline \multicolumn{6}{|c|}{ g. Vertebrae number } \\
\hline Counts & $15 / 12$ & $15 / 13$ & & & \\
\hline Specimens & 2 & 1 & & & \\
\hline
\end{tabular}

kyar, B. pyema and B. ruberare known from Irrawaddy drainage (Kullander \& Britz 2002). The new species differs from B. kyar, B. corycaeus B. pyema in the presence (vs. absence) of cleithral blotch; from $B$. ruber in absence (vs. presence) of a dark black blotch on dorsolateral aspect of caudal peduncle. It also differs from $B$. ferrarisi in absence (vs. presence) of the vertical bars across side and caudal peduncle.

Badis dibruensis also differs from $B$. siamensis and $B$. khwae respectively of Takaupa River basin and Mae Nam Khwae Noi drainage, Thailand in absence (vs. presence) of a dark blotch on dorso-lateral aspect of caudal peduncle; from

Table 4. Comparison of proportional measurements in percentage of standard length and counts of Badis dibruensis sp. nov. with related species in mean and (range in parentheses).

\begin{tabular}{|c|c|c|c|c|}
\hline Proportions & B. dibruensis sp. nov. & B. badis & B. kanabos & B. tuivaiei \\
\hline Interorbital width & $11.4(9.9-15.0)$ & $7.4(6.5-8.3)$ & $5.7(5.1-6.2)$ & $6.4(5.6-7.2)$ \\
\hline Orbital diameter & $8.6(7.6-9.4)$ & $11.6(10.8-12.3)$ & $11.1(9.5-12.7)$ & $7.5(6.1-9.4)$ \\
\hline Upper jaw length & $6.4(6.1-6.9)$ & $9.2(8.2-10.2)$ & $8.7(7.6-9.8)$ & $8.9(8.5-10.4)$ \\
\hline Lower jaw length & $7.8(7.1-8.3)$ & $12.9(11.3-14.5)$ & $10.2(9.8-10.5)$ & $13.9(10.9-16.4)$ \\
\hline Vertebrae & 27 & $27(26-28)$ & $27(26-28)$ & $31(30-31)$ \\
\hline
\end{tabular}

Key to species of genus Badis of India

$\begin{array}{ll}\text { 1. } & \text { Body without dark brown or vertical black bars on sides } \\ \text { Body with dark brown or vertical black bars on side } \\ \text { Conspicuous blotch behind opercle dorsally } \\ \text { No blotch behind opercle dorsally } \\ \text { Body with } 10-11 \text { distinct bars } \\ \text { Body with indistinct bars } \\ \text { Toothplates } 7-9, \text { scales in lateral row } 29 \\ \text { Toothplates more than } 9 \text {, scales in lateral row } 27 \\ \text { No spot between } 3-5 \text { dorsal fin spines } \\ \text { A dark spot between } 3-5 \text { dorsal fin spines } \\ \text { Bars narrow, circumpeduncular scales } 16\end{array}$




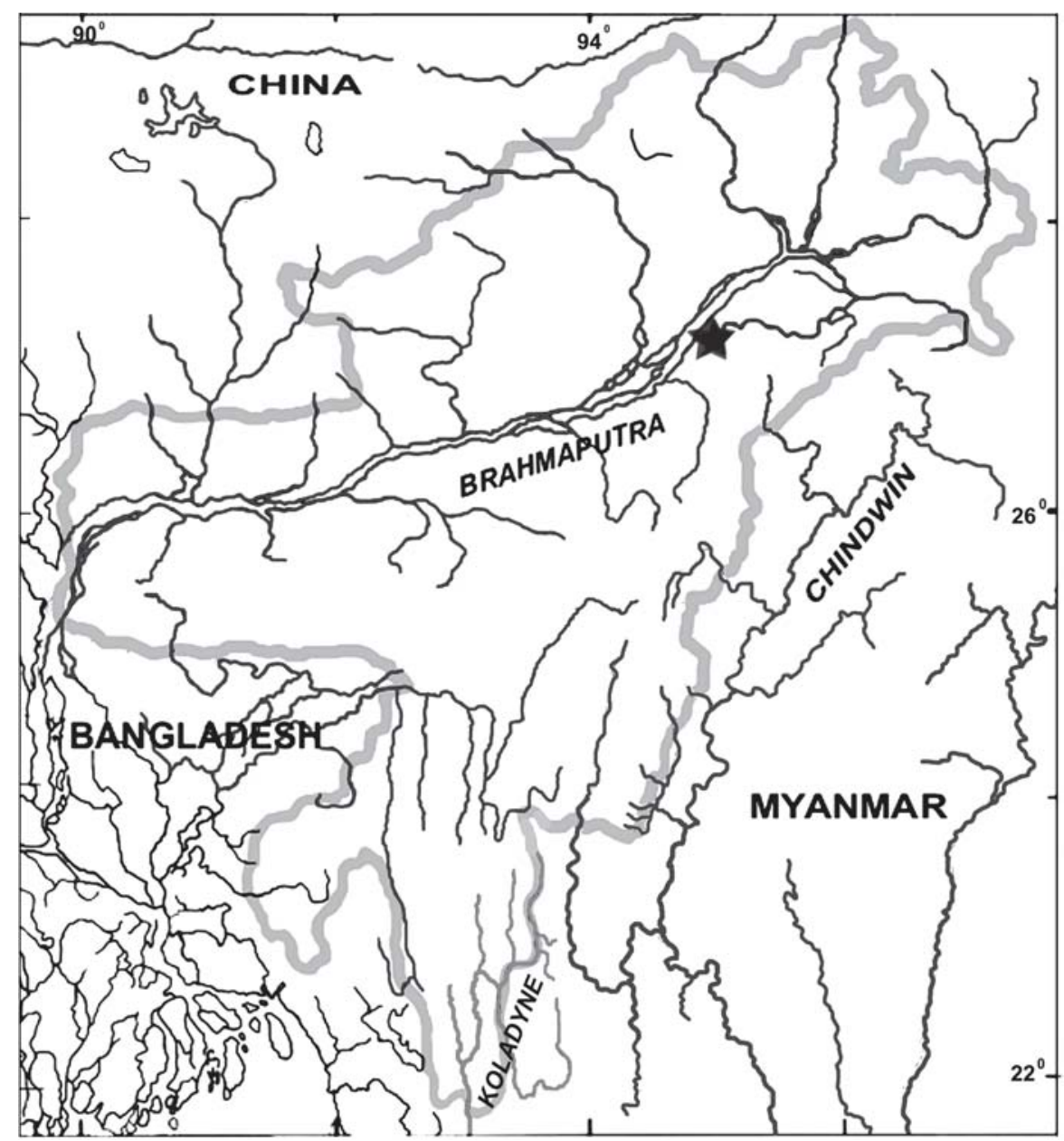

Figure 1. Map showing type locality of Badis dibruensis sp. nov.

B. chittagongis of Matamohuri River drainage, Bangladesh, in absence (vs. presence) of the vertical bars across side and caudal peduncle.

Kullander \& Britz (2002) classified the species of Badis into five groups viz., B. ruber group, B. assamensis group, $B$. corycaeus group, $B$. kyar group and $B$. badis group. The species of $B$. badis group share a cleithral blotch. They expected more number of species in $B$. badis group from the view i.e. the limited material from India, variable characters and scattered locality record of the species. The present species under diagnosis belongs to $B$. badis group.

Out of the known 13 valid species of Badis, six is reported from northeast India. In addition to this the present species under study is also from the Brahmaputra basin. General inventory and phylogenetic study of the diverse species of Badis in northeast India and adjoining areas would be of great interest.

\section{Comparative materials}

Badis assamensis: MUMF Per-51-54, 4, 41.6-55.8 mm SL, Dibru River, Dibrugarh, Assam, India; B. badis: MUMF Per-5565, 11, 23.5-28.7 mm SL, Barak River, Manipur, India; $B$. blosyrus: MUMF Per-66-68, 3, 36.8-38.9 mm SL, Teju River, Teju District, Arunachal Pradesh, India; $B$. ferrarisi: MUMF Per69-75, 7, 32.0-44.0 mm SL, Lokchao River, Manipur, India; $B$. kanabos: MUMF Per-76-81, 6, 48.7-54.9 mm SL, Barak River; Manipur, India; B. tuivaiei: MUMF 5125-5132, 8, 53.5-59.4 mm SL, Tuivai and Irang River, Manipur, India.

\section{References}

AhI, E. (1937). Neue susswasserfische aus dem Indischen und Malaiischen Gebiet. Zoologischer Anzeiger 117: 113-119.

Barlow, G.W., K.F. Leim \& W. Wickler (1968). Badidae, a new fish family- behavioural, osteological, and development evidence. Journal of Zoology 156: 415-447.

Bleeker, P. (1854). Nalezingen op de ichthyologische fauna van Bengalen en Hindostan. Verhandeligen van het Bataviaasch Genootschap van Kunsten en Wettenschappen 25(8): 1-166.

Greenwood, P.H. (1976). A review of the family centropomidae (Pisces, Perciformes). Bulletin of the British Museum (Natural History) 29(1): 1-81.

Hamilton, F. (1822). An Account of the Fishes found in the River Ganges and Its Branches. Archibald Constable and Company, London, 405pp + 39pls.

Hollister, G. (1934). Clearing and dyeing fish for bone study. Zoologica 12: 89-101.

Kullander, S.O. \& R. Britz (2002). Revision of the family Badidae (Teleostei: Perciformes), with description of a new genus and ten new species. Ichthyological Exploration of Freshwaters 13(4): 295-372.

Talwar, P.K. \& A.G. Jhingran (1991). Inland Fishes of India and Adjacent Countries. Oxford and IBH Publishing Co. Pvt. Ltd., New Delhi, 2 volumes, xix + 1158pp.

Vishwanath, W. \& K. Shanta (2004). A new fish species of the IndoBurmese genus Badis Bleeker (Teleostei: Perciformes) from Manipur, India. Zoos' Print Journal 19(9):1619-1621.

Vishwanath, W., W.S. Lakra \& U.K. Sarkar (2007). Fishes of North East India. National Bureau of Fish Genetic Resources, Lucknow, UP, India, 264pp. 